\title{
Factores ambientales controladores del fitoplancton con énfasis en las cianobacterias potencialmente tóxicas en un lago somero utilizado como fuente de agua para potabilización: Laguna del Sauce, Maldonado, Uruguay
}

\author{
Environmental drivers of phytoplankton with focus \\ on potentially toxic cyanobacteria in a shallow lake used \\ as drinking water source: Sauce Lake, Maldonado, Uruguay
}

González-Madina, Lucía (1); Pacheco, Juan Pablo (1); Mazzeo, Néstor (1,2,4); Levrini, Paula (1); Clemente, Juan María (1); Lagomarsino, Juan José (3); Fosalba, Claudia (1).

(1) Departamento de Ecología y Gestión Ambiental, Centro Universitario Regional del Este (CURE), Universidad de la República

(UdelaR), Uruguay.

(2) Instituto SARAS, Uruguay.

(3) Área Hidrobiología de la Gerencia de Gestión de Laboratorios, Obras Sanitarias del Estado, (OSE-UGD), Uruguay.

(4) Facultad de Ciencias, Universidad de la República (UdelaR), Uruguay.

Contacto: luciagm17@gmail.com

RECIBIDO: 6/4/2017 - APROBADO: 10/6/2017

\begin{abstract}
Resumen
Laguna del Sauce, principal fuente de agua para potabilización en Maldonado, Uruguay, ha registrado recurrentes eventos de floraciones de cianobacterias potencialmente tóxicas, que comprometen su uso como tal. Esto ha denotado la necesidad de un mayor conocimiento de los factores ambientales asociados a la composición fitoplanctónica y, en especial, a la ocurrencia de floraciones de cianobacterias. Mediante muestreos semanales estivales (2015-2016) de variables fisicoquímicas, nutrientes y fitoplancton, en seis sitios de Laguna del Sauce, se analizaron los factores ambientales asociados a la biomasa y composición fitoplanctónica, particularmente de cianobacterias. La alta variabilidad temporal y espacial observada en la composición fitoplanctónica presentó alternancia de floraciones de cianobacterias: Dolichospermum crassum, Cuspidothrix issatschenkoii, Microcystis aeruginosa, Microcystis panniformis y Sphaerocavum brasiliense. La turbiedad fue clave para regular la biomasa fitoplanctónica, mientras que la relación Nitrógeno total (NT):Ortofosfato (PO4) se asoció a la presencia diferencial de especies de cianobacterias. El análisis de las relaciones entre estos nutrientes puede contribuir a anticipar la ocurrencia de floraciones de diferentes especies de cianobacterias en este ecosistema, posibilitando la implementación de medidas de gestión orientadas a disminuir sus efectos adversos y, en particular, a asegurar el proceso de potabilización.

Palabras clave: Floraciones nocivas, composición fitoplanctónica, biomonitoreo, aprovisionamiento de agua.
\end{abstract}

Abstract

Sauce Lake, the main drinking water source in Maldonado, Uruguay, has been registering recurring events of potentially toxic cyanobacterial blooms that affect its service. This leads to the necessity of exploring the environmental drivers in the composition of phytoplankton, particularly in the presence of potentially toxic cyanobacteria. In this study we analyzed the environmental factors associated with phytoplankton composition and biomass, particularly of cyanobacteria. We sampled physicochemical variables, nutrients and phytoplankton during the summer 2015-2016 with a weekly frequency, in six sites of Sauce Lake. The results show a very high temporal and spatial variability in the phytoplankton composition, with cyanobacterial algal blooms of different taxa: Dolichospermum crassum, Cuspidothrix issatschenkoi, Microcystis aeruginosa, Microcystis panniformis y Sphaerocavum brasiliense. We observed that turbidity is a key factor controlling the total phytoplankton biomass, while the NT vs. PO4 concentrations determined the differential presence of potentially toxic cyanobacteria. The analysis of the relationship between these nutrients could contribute to anticipate different cyanobacterial harmful algal blooms in this ecosystem, enabling the development of management tools oriented to decrease its negative effects, particularly in the purification process.

Keywords: Harmful Algal blooms, phytoplankton composition, biomonitoring, drinking water supply. 


\section{Introducción}

El aumento en la carga de nutrientes en ecosistemas acuáticos, conocido como proceso de eutrofización (Hutchinson, 1973; Wetzel, 2001; Prepas y Charette, 2003), constituye la principal afectación de los ecosistemas de agua dulce a nivel mundial (Wetzel, 2001; Khan y Ansari, 2005; Smolders, et al., 2006). Con el avance de este proceso surgen diversos efectos en el ecosistema, como la disminución de la transparencia, los cambios abruptos en la concentración de oxígeno disuelto y su eventual agotamiento temporal, así como el incremento de la biomasa de los productores primarios (Khan y Ansari, 2005; Schindler, 2006; Smolders, et al., 2006). En estados avanzados de este proceso, una de las principales consecuencias es el aumento de la biomasa de fitoplancton en general, y en particular el aumento en la frecuencia y persistencia temporal de altas biomasas de cianobacterias, eventos conocidos como floraciones (Tarczynska, et al., 2001; Khan y Ansari, 2005; Paerl, et al., 2011). Una característica de particular relevancia de las floraciones de cianobacterias es su capacidad de producción de toxinas (cianotoxinas) (Carmichael, 1992; Carmichael, 1997; Chorus, 2012). La producción de cianotoxinas depende de la composición taxonómica de las cianobacterias presentes en la floración, algunas cianotoxinas son características de determinados géneros (por ejemplo, microcystinas mayormente presentes en los géneros Microcystis, Planktolyngbya y Dolichospermum; cylindrospermopsina en Cylindrospermopsis y Aphanizomenon; anatoxina en Aphanizomenon y Dolichospermum) (Merel, et al., 2013; De León, 2002; Bonilla, 2009). La producción de estos compuestos tóxicos, así como de otros metabolitos secundarios que pueden conferir olor-sabor al agua, pueden coexistir en la misma floración y estar influidos por parámetros ambientales como la luz, la temperatura o los nutrientes disponibles (Dolman, et al., 2012; Paerl y Otten, 2013).

Estas y otras consecuencias derivadas de la presencia de cianobacterias pueden interferir negativamente con los múltiples usos y servicios que los ecosistemas acuáticos proveen, como la recreación, pesca, navegación y el suministro de agua potable, entre otros (Khan y Ansari, 2005; Stewart, et al., 2006; Carpenter, et al., 2011). Particularmente, las floraciones de cianobacterias afectan negativamente la capacidad de los ecosistemas como fuente de abastecimiento de agua para potabilización (Falconer, et al., 1994; Falconer y Humpage, 2005). Los inconvenientes sanitarios derivados de su toxicidad o producción de metabolitos vinculados a olor-sabor en agua implican regularmente elevados costos económicos para las poblaciones humanas, y pueden desencadenar diversos impactos sociales (Chorus y Bartram, 1999; WHO, 2004).

La presencia de floraciones de cianobacterias en ecosistemas acuáticos con altos niveles de nutrientes se debe, en parte, a que constituyen un grupo altamente diverso, con una amplia variedad de atributos morfológicos y funcionales que determinan su capacidad de adaptación diferencial y dominancia en los ensambles fitoplanctónicos de sistemas eutróficos (Robarts y Zohary, 1987; Smith y Bennett, 1999; Schindler, 2006; Heisler, et al., 2008; Paerl y Otten, 2013). Entre los atributos presentes en las cianobacterias, la capacidad de fijación de nitrógeno inorgánico atmosférico es frecuentemente destacada como uno de los de mayor relevancia ecológica (Reynolds, 1987; Paerl, 1997; Smith y Bennett, 1999; Reynolds, 2006; Paerl y Otten, 2013). En condiciones de baja disponibilidad relativa de nitrógeno, y cuando otros nutrientes no resultan limitantes, las cianobacterias presentan una ventaja adaptativa de incorporación de nutrientes con respecto a otros grupos (Ferber, et al., 2004; Schindler, et al., 2008; Dolman, et al., 2012; Paerl y Otten, 2013). Dada esta capacidad de fijación en condiciones de baja disponibilidad de $\mathrm{N}$ en relación al $\mathrm{P}$, y que por lo general en los lagos eutróficos existe una sobredisponibilidad de $\mathrm{P}$ en relación al $\mathrm{N}$, clásicamente la mayor parte de los esfuerzos para limitar el desarrollo de cianobacterias en ecosistemas acuáticos se ha enfocado en la disminución de la carga interna de fósforo (Moss, et al., 1996; Conley, et al., 2009).

El estudio de los factores ambientales asociados a la composición fitoplanctónica y, en particular, de las condicionantes ambientales que influyen en la aparición y persistencia de floraciones de cianobacterias es crucial para el entendimiento del funcionamiento ecosistémico y especialmente para evitar o mitigar los efectos adversos generados por estas floraciones.

Recientemente, en la región este de Uruguay se han evidenciado importantes eventos de floraciones de cianobacterias potencialmente tóxicas (Vidal y Kruk, 2008; Pacheco, et al., 2010; Bonilla, et al., 2015), algunas de las cuales se han vinculado a graves afectaciones de la calidad de agua (Mazzeo, et al., 2010; Pacheco, et al., 2010; Bonilla, et al., 2015). En particular, Laguna del Sauce (Maldonado, Uruguay), la principal fuente de agua para potabilización de la región este de Uruguay, ha visto comprometida su aptitud para tal uso debido al aumento en la recurrencia y persistencia temporal de floraciones de cianobacterias (Rodríguez, et al., 2010; Mazzeo, et al., 2010; Crisci, et al., en prensa). Estas consecuencias del proceso de eutrofización del ecosistema se han visto magnificadas por el aumento de la actividad urbana y agrícola en la cuenca de drenaje, con el consecuente aumento del ingreso de nutrientes al sistema, así como por un aumento en el tiempo de residencia por represamiento de este ecosistema (Mazzeo, et al., 2010). Laguna del Sauce posee una gran heterogeneidad espacial, así como una alta variabilidad temporal asociada a la biomasa y composición fitoplanctónica (Mazzeo, et al., 2010; Pacheco, et al., 2016). En este sentido, Crisci et al. (en prensa) describen un rol clave de la turbiedad inorgánica como limitante de la producción fitoplanctónica y observan que a niveles de turbiedad superiores a 30 NTU no es posible el desarrollo de altas biomasas fitoplanctónicas como las presentes en periodos de floraciones de cianobacterias. Estos períodos de alta turbiedad son desencadenados por eventos de vientos fuertes, lo que confiere al sistema una alta variabilidad temporal respecto a la biomasa fitoplanctónica (Crisci, et al., en prensa). Esta condición, acoplada a la frecuente aparición de floraciones de cianobacterias, puede comprometer en gran medida la capacidad de este ecosistema de aprovisionamiento de agua para potabilización (Mazzeo, et al., 2010; Pacheco, et al., 2016; Crisci, et al., en prensa). Por esto, el análisis de los factores ambientales que se asocian a diferentes biomasas y composiciones de fitoplancton, y en particular de las floraciones de cianobacterias, son de fundamental relevancia para el conocimiento ecosistémico en general y para la capacidad de anticiparse al desarrollo de estas floraciones, lo que permite adoptar medidas de manejo que minimicen sus efectos adversos. En este trabajo se analizan los principales factores ambientales vinculados a la biomasa y composición fitoplanctónica, con particular énfasis en aquellos asociados a la presencia de cianobacterias potencialmente tóxicas, durante un periodo estival en Laguna del Sauce, considerando su uso como fuente de agua para potabilización. 


\section{Materiales y Métodos}

\section{Área de estudio}

La Laguna del Sauce ( $34^{\circ} 43^{\prime}$ S, $5^{\circ} 13^{\prime} \mathrm{W}$ ) es un sistema eutrófico de gran tamaño (12 km largo máximo y $3,5 \mathrm{~km}$ de ancho promedio) ubicado en la costa del departamento de Maldonado en la región este de Uruguay (Figura 1).

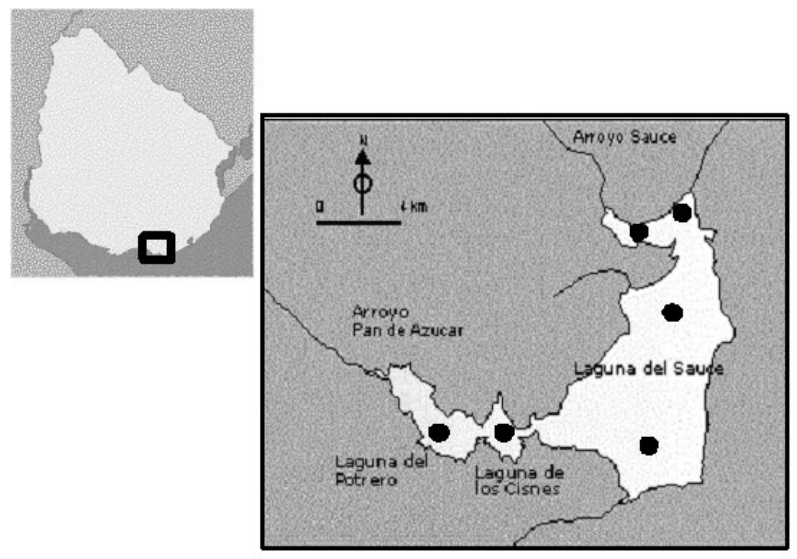

Figura 1. Ubicación de la Laguna del Sauce y los puntos de muestreo en el sistema principal y subsistemas asociados. Puntos: 1) Arroyo Sauce; 2) Bahía Mallorquina; 3) Sauce Norte; 4) Sauce Sur; 5) Laguna De los Cisnes; 6) Laguna del Potrero.

Está formado por tres lagos someros conectados de manera permanente: del Sauce (sistema principal, 4045 ha), Cisnes (205 ha) y Potrero (411 ha). Estos dos últimos junto con uno de sus principales tributarios, Arroyo Sauce, se definen como los tres subsistemas asociados al sistema principal (Laguna del Sauce). Por su escasa profundidad (media $=2,5 \mathrm{~m}$; máxima $=5 \mathrm{~m}$ ) y la dirección de su largo máximo efectivo perpendicular a la línea de costa, este sistema es fuertemente influenciado por los vientos y genera una gran resuspensión de los sedimentos que llevan a estados de alta turbiedad inorgánica en el sistema.

El sistema ha sido clasificado como eutrófico, con valores de fósforo total en el rango de 80-100 $\mu$ g.L. $\mathrm{L}^{-1}$ y de nitrógeno total de 200-1200 $\mu$ g. $L^{-1}$ (Inda y Steffen, 2010), $\mathrm{y}$ en los últimos años se han intensificado los aportes de nutrientes que ingresan desde su cuenca (Mazzeo, et al., 2010). Este incremento, resultado de la intensificación agrícola así como del aumento en la urbanización y la actividad turística durante las últimas décadas, ha repercutido en un aumento en la frecuencia de aparición y persistencia de floraciones de cianobacterias (Mazzeo, et al., 2010; Rodríguez, et al., 2010).

Laguna del Sauce es actualmente considerada la segunda fuente de agua para potabilización en Uruguay y la principal para la región este, en cantidad de población abastecida (aproximadamente 160.000 habitantes permanentes y más del doble de turistas durante la temporada estival) (Inda y Steffen, 2010), desde la planta potabilizadora de OSE-UGD situada en el margen este de la laguna.

\section{Colecta y análisis de muestras fisicoquímicas y biológicas}

En este estudio se abarcó un período de muestreo comprendido entre diciembre de 2015 y abril de 2016, en el cual se realizaron colectas semanales en el sistema principal de Laguna del Sauce: puntos correspondientes a Bahía de la Mallorquina, Sauce Norte y Sauce Sur; y sistemas asociados: Laguna De los Cisnes, Laguna del Potrero y Arroyo Sauce (Figura 1).

En cada uno de estos puntos se midió la penetración lumínica mediante disco de Secchi (SD), se realizaron perfiles de luz en profundidad mediante irradiómetro (Li-cor $4 \mathrm{pi}$ ) y se midieron los principales parámetros ambientales in situ: temperatura (T), conductividad (Cond), turbiedad (NTU), potencial óxido-reducción (ORP), oxígeno disuelto (OD y $\% \mathrm{OD}$ ) y ficocianina (PC) mediante sonda multiparamétrica YSI 6600. En cada punto de muestreo se tomaron muestras de la columna de agua integradas en profundidad por triplicado, mediante tubo muestreador de $10 \mathrm{~cm}$ de diámetro y $2 \mathrm{~m}$ de altura. Una fracción de este integrado, conservada en frío y oscuridad, fue destinada para los análisis fisicoquímicos y de nutrientes en laboratorio: sólidos totales en suspensión (SST por secado a $105^{\circ} \mathrm{C}$ por 12 horas), materia orgánica (MO, por diferencia de peso post quemado a $500{ }^{\circ} \mathrm{C}$ ), nutrientes: nitrógeno total (NT; Koroleff, 1970), amonio (NH4; Muller, 1955), nitratos (NO3), fósforo total (PT; Valderrama, 1981), ortofosfato (PO4; Murphy, 1962), clorofila_a (Chl_a por extracción en etanol frío y fluorometría; Nusch, 1980).

Otra fracción de este integrado por sitio se fijó en lugol acidificado y fue destinada a la cuantificación del fitoplancton en laboratorio mediante conteos en cámaras de sedimentación de 10 o $20 \mathrm{~mL}$ en microscopio invertido a $400 \mathrm{X}-1000 \mathrm{X}$ (Utermöhl, 1958; Guillard, 1978), en campos al azar (Uhelinger, 1964) y considerando como mínimo 100 organismos de la especie más frecuente (Lund, et al., 1958).

Las muestras cualitativas de fitoplancton, destinadas a la identificación y medición de los organismos, fueron tomadas mediante arrastres con red de plancton de 20 micras de malla y fijadas en Formaldehído acidificado (Formol 40 \% + mismo Vol. Ac. Acético) hasta su tratamiento en laboratorio. A partir de estas muestras se realizó la medición, identificación taxonómica y fotografiado de los organismos, colonias o cenobios fitoplanctónicos mayores a 2 micras, en microscopio directo Nikon Eclipse 50 i a 400X y 1000X aumentos.

Para la identificación taxonómica fueron utilizadas claves internacionales y regionales. Para la medición fue utilizada una cámara CMOS 5.0 Mpx y el software Micrometrics ${ }^{\circ}$ Premium Edition, previamente calibrado para los diferentes aumentos utilizados. El volumen individual de los organismos fue calculado considerando su forma geométrica según Hillebrand et al. (1999), midiendo al menos 30 organismos por cada especie.

\section{Procesamiento y análisis de datos}

Los patrones temporales de biomasa y composición de fitoplancton por punto de muestreo fueron inspeccionados visualmente a partir de la representación gráfica de los datos. Posteriormente, se analizó gráficamente el patrón temporal en la biomasa de cianobacterias, en particular en relación a los niveles de alerta de agua para potabilización según la normativa interna de OSE (Gravier, et al., 2017). Estos niveles de alerta, referidos al agua bruta (tomada de la fuente 
pero previa al tratamiento), establecen diferentes alertas según el biovolumen de cianobacterias. Se define estado de "Vigilancia» cuando se estima un biovolumen entre 0,05 y $0,2 \mathrm{~mm}^{3}: \mathrm{L}^{-1}$, estado de «Alerta 1 » cuando se estima un biovolumen entre 0,2 y $1,0 \mathrm{~mm}^{3} . \mathrm{L}^{-1}$, estado de «Alerta 2» cuando se estima un biovolumen entre 1,0 y $2,0 \mathrm{~mm}^{3} \cdot \mathrm{L}^{-1}$ ) y estado de «Alerta 3 » cuando se estima un biovolumen $\geq 2,0$ $\mathrm{mm}^{3}$. $^{-1}$ (Gravier, et al., 2017). A pesar de que estos niveles de alerta son generalmente utilizados para agua bruta, en este estudio fueron utilizados como referencia para el agua del sistema fuente.

Con el fin de analizar qué variables ambientales fueron las que explicaron mayormente la composición fitoplanctónica, se realizó un análisis de correspondencia canónica (CCA), considerando las variables fisicoquímicas como variables explicatorias y el biovolumen de las especies (presentes en biovolúmenes mayores a $5 \%$ en la muestra) logaritmizado $(\log (\mathrm{x}+1)$ como variable de respuesta. La significancia del modelo completo del CCA fue testeada mediante permutaciones de Monte Carlo con 999 ordenaciones irrestrictas. Asimismo, fueron testeadas la significancia de los ejes canónicos de ordenación y de cada variable incluida en el modelo, asumiendo un p valor máximo de 0,05 en cada caso.

De las variables ambientales que mejor explicaron la composición fitoplanctónica, fueron seleccionadas el NT y PO4 como aquellas con mayor capacidad predictiva y por constituir descriptores básicos del estado trófico. Fueron realizadas regresiones lineales simples para conocer la relación entre NT y PO4 en relación a la biomasa de cianobacterias, estimada mediante la concentración de ficocianina (PC). Para esto fue previamente testeada la normalidad de los datos mediante test de bondad de ajuste de Kolmogorov-Smirnov.

Asimismo, fueron evaluados los patrones de respuesta de las diferentes especies de cianobacterias registradas (en biovolúmenes mayores a $5 \%$ ) a las concentraciones de NT y PO4 mediante representación gráfica del biovolumen (gráfico de mapa de calor).

Todos los análisis estadísticos fueron realizados en $\mathrm{R}$ mediante el paquete «vegan» (R-Core team 2016) y la representación gráfica fue realizada en Sigmaplot 12.0 (Systat software Inc.).

\section{Resultados}

Se observó una gran variabilidad temporal y espacial en las abundancias de fitoplancton para los diferentes puntos de muestreo (Figura 2). Los eventos de altos biovolúmenes de fitoplancton fueron observados exclusivamente en los puntos de muestreo pertenecientes al sistema principal de Laguna del Sauce (Bahía Mallorquina, Sauce Norte, Sauce Sur) (Figura 2), que presentaron altos niveles de clorofila y ficocianina (Tabla 1). La composición de fitoplancton en el sistema principal estuvo dominada fundamentalmente por Dinophyta y cianobacterias, desde diciembre de 2015 hasta finales de febrero de 2016. En este período se registraron los mayores biovolúmenes de cianobacterias asociados a floraciones (Figuras 2 y 3). En los sistemas asociados (Arroyo Sauce, Cisnes y Potrero) la composición del fitoplancton fue más variada, con dominancia de grupos como Euglenozoa, Chlorophyta, Dinophyta a lo largo del periodo de muestreo (Figura 2).

La composición fitoplanctónica de los puntos de muestreo pertenecientes al sistema principal de la Laguna del Sauce mostró un claro recambio a modo de periodos: el primero dominado por cianobacterias filamentosas del orden Nostocales, fundamentalmente Dolichospermum crassum, con rápido recambio a la especie Cuspidothrix issatschenkoi (acompañada de filamentos del género Aphanizomenon), el segundo dominado por cianobacterias chroococcales (colonias de las especies Microcystis aeruginosa, Microcystis panniformis y Sphaerocavum brasiliense), y el último dominado por dinoflagelados (principalmente de la especie Ceratium furcoides), criptofitas y diatomeas (Figuras $2 \mathrm{y}$ 3). Las altas biomasas de cianobacterias se restringieron de manera exclusiva al cuerpo de agua principal y al periodo temporal de diciembre de 2015 a comienzos de marzo de 2016. Durante este periodo, los eventos de floraciones de cianobacterias observados en el sistema principal de Laguna del Sauce mostraron una rápida alternancia entre situaciones de biomasas muy altas a la caída abrupta en las abundancias de fitoplancton en intervalos de una semana (Figura 2). En particular, estos periodos de mayor biovolumen de fitoplancton estuvieron asociados a la presencia de floraciones de cianobacterias potencialmente tóxicas, fundamentalmente de Dolichospermum crassum, Microcystis aeruginosa y Aphanizomenon spp. y Cuspidothrix issatschenkoii (Figura 3) en conjunto con Ceratium furcoides (Dinophyta). Todos estos eventos de floraciones de cianobacterias, que persistieron hasta comienzos de marzo, presentaron biovolúmenes de entre 5 y $35 \mathrm{~mm}^{3} \cdot \mathrm{L}^{-1}$, los cuales son sumamente superiores al límite establecido por el máximo nivel de alerta de calidad de agua para potabilización estipulado por la normativa de OSE (Gravier, et al., 2017).

\begin{tabular}{|c|c|c|c|c|c|c|c|c|c|c|c|c|}
\hline & $\mathrm{T}$ & NT & NO3 & NH4 & PT & PO4 & SST & MO & Chl_a & DS & Turb & Ficocian \\
\hline & $\left({ }^{\circ} \mathrm{C}\right)$ & $\left(\mu \mathrm{g} . \mathrm{l}^{-1}\right)$ & $\left(\mu \mathrm{g} . \mathrm{l}^{-1}\right)$ & $\left(\mu \mathrm{g} . \mathrm{l}^{-1}\right)$ & $\left(\mu \mathrm{g} . \mathrm{l}^{-1}\right)$ & $\left(\mu \mathrm{g} . \mathrm{l}^{-1}\right)$ & $\left(\mathrm{mg} \cdot{ }^{-1}\right)$ & (\%) & $\left(\mu \mathrm{g} . \mathrm{l}^{-1}\right)$ & $(\mathrm{cm})$ & (NTU) & $\left(\right.$ cel.ml $\left.{ }^{-1}\right)$ \\
\hline Máx & 27,4 & 1302,4 & 250,9 & 804,6 & 125,4 & 61,5 & 29,0 & 100 & 78,9 & 110 & 30,6 & 23288 \\
\hline Mín & 20,7 & 505,9 & 103,8 & 21,4 & 48,4 & 2,9 & 3,5 & 12 & 2,2 & 45 & 5,0 & 1109 \\
\hline Promedio & 24,3 & 848,9 & 166,8 & 153,9 & 83,4 & 22,2 & 10,6 & 59 & 28,1 & 63,8 & 16,6 & 7028 \\
\hline
\end{tabular}

Tabla 1. Principales parámetros fisicoquímicos para Laguna del Sauce en el periodo estival 2015-2016. Valores máximos, mínimos y promedio de: temperatura (T), nitrógeno total (NT), nitrato (NO3), amonio (NH4), fósforo total (PT), fosfato (PO4), sólidos totales en suspensión (SST), materia orgánica (MO), clorofila a (Chl_a), profundidad de disco de secchi (DS), turbiedad (Turb) y ficocianina (Ficocian). 

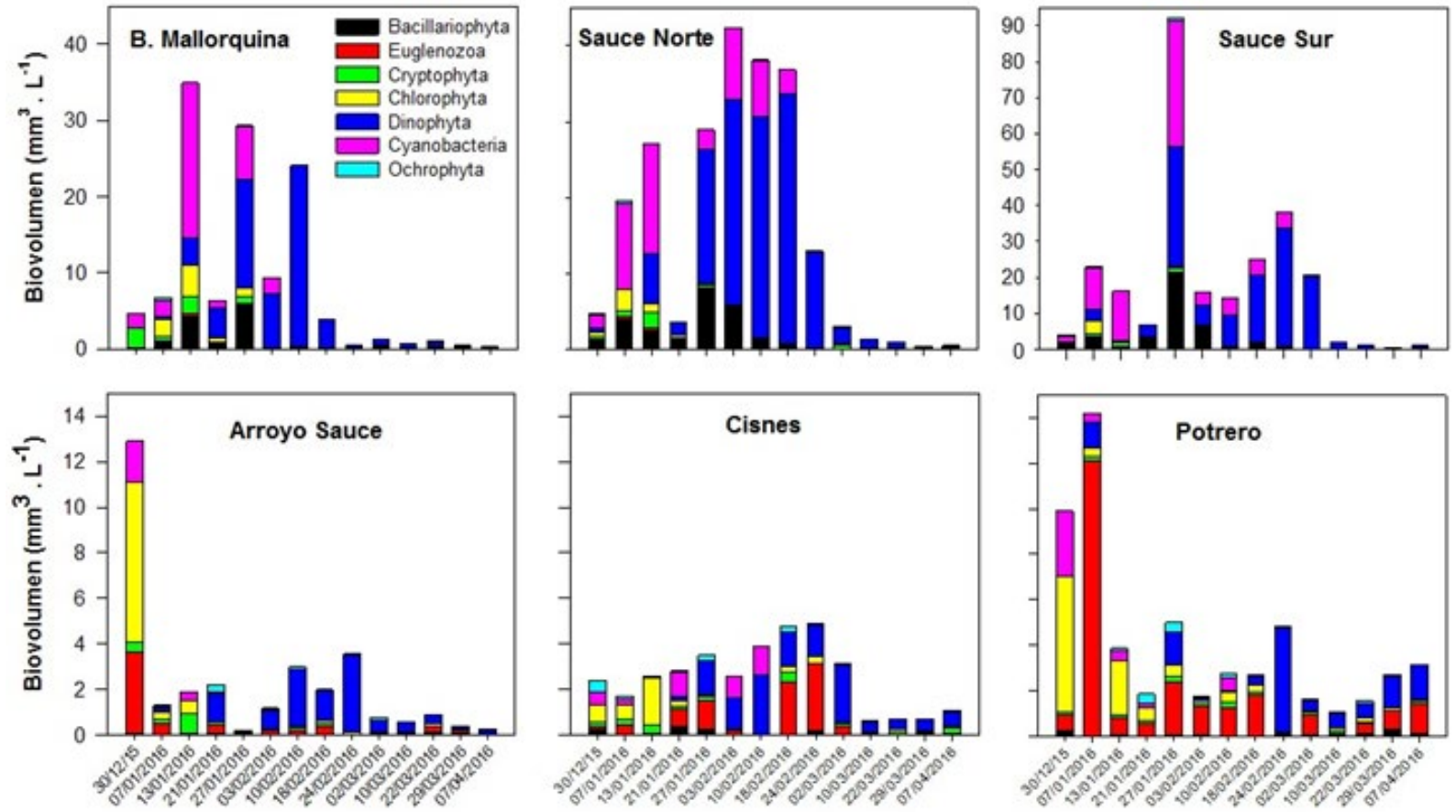

Figura 2. Variación temporal en la composición de fitoplancton para cada sitio de muestreo en Laguna del Sauce. Período: Diciembre 2015 - Abril 2016. Nótese la diferencia de escalas en el eje Y en los gráficos superiores.

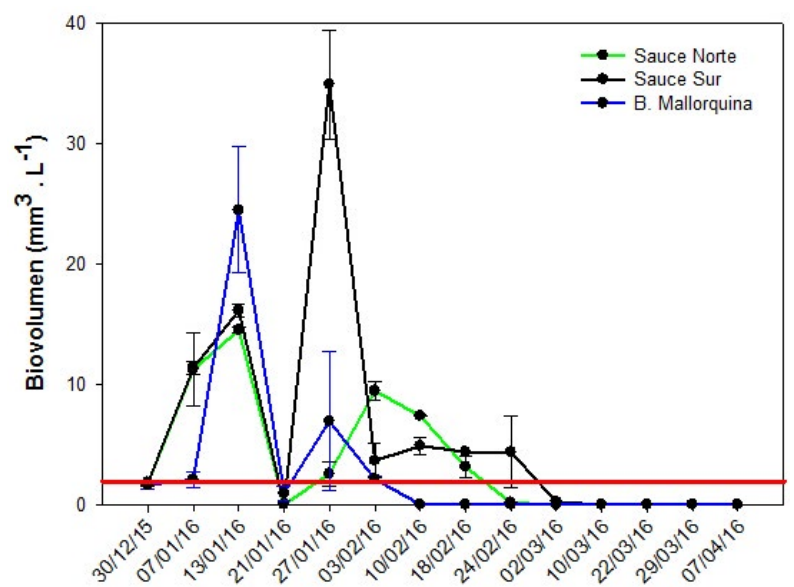

Figura 3. Variación del biovolumen de cianobacterias en el cuerpo de agua principal de la Laguna del Sauce para las fechas de muestreo consideradas. La línea horizontal roja representa el biovolumen correspondiente a un nivel de alerta 3, considerando agua bruta para potabilización.

En cuanto a las variables ambientales asociadas a la composición fitoplanctónica, el análisis de correspondencia canónica (Figura 4) indicó que el ortofosfato (PO4), el nitrógeno total (NT), la penetración lumínica (DS), los sólidos en suspensión (SST), la turbiedad (NTU) y la temperatura del agua $(\mathrm{T})$ fueron los principales factores que explicaron la variabilidad en la composición del fitoplancton, explicando el $61,2 \%$ de la varianza total $\left(\lambda_{1}=20,4\right.$ y $\left.\lambda_{2}=15,92\right)$ (significancia del modelo de $\mathrm{F}=1,89$ y $\mathrm{p}<0,05$; significancia de

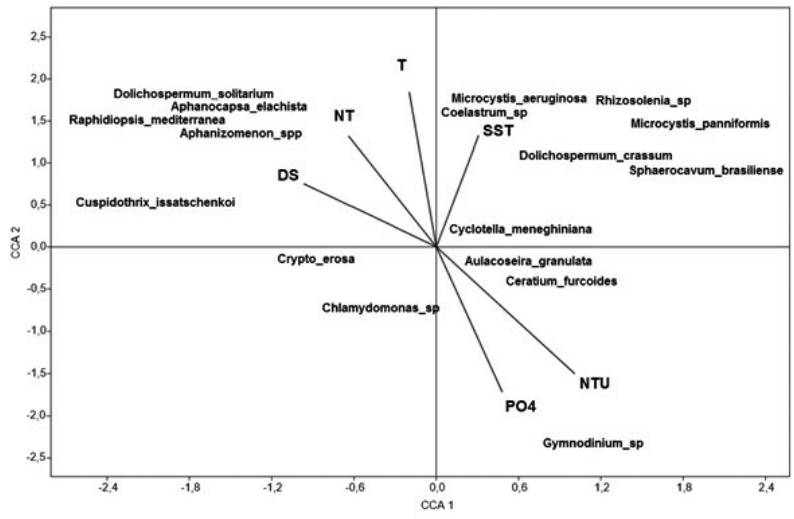

Figura 4. Biplot del análisis de correspondencia canónica de las variables ambientales asociadas a la composición fitoplanctónica. Las flechas representan las variables ambientales consideradas: DS= profundidad de disco de Secchi, $\mathrm{NT}=$ nitrógeno total, $\mathrm{T}=$ temperatura, $\mathrm{SST}=$ sólidos suspendidos totales, $\mathrm{NTU}=$ turbiedad, $\mathrm{PO} 4=$ fosfato.

ejes: CCA1 : $\mathrm{F}=4,26$ y $\mathrm{p}<0,01, \mathrm{CCA} 2: \mathrm{F}=3,32$ y $\mathrm{p}<0,01)$. Dentro de las variables explicativas, el fosfato resultó más significativo $(\mathrm{F}=3,13 \mathrm{y} \mathrm{p}<0,05)$.

Las especies de cianobacterias Chroococcales (Microcysits aeruginosa, Microcystis panniformis y Sphaerocavum brasiliense), así como la especie de cianobacteria nostocal Dolichospermum crassum se asociaron positivamente con el nitrógeno total (NT), los sólidos totales disueltos (SST) y con la temperatura $(\mathrm{T})$, pero negativamente con la turbiedad 
(NTU) y el fosfato (PO4). A su vez, el resto de las especies de cianobacterias identificadas, Cuspidothrix issatschenkoi, Dolichospermum solitarium, Raphidiopsis mediterranea y Aphanizomenon sp, se asociaron positivamente con la profundidad de disco de secchi (DS) y con el nitrógeno total (NT), pero también negativamente con la turbiedad (NTU) y con el fosfato (PO4) (Figura 4). En este sentido, se observó que los factores más importantes que explican la biomasa de cianobacterias son el nitrógeno total (NT), el ortofosfato (PO4) y la turbiedad (NTU). Considerando exclusivamente NT y NTU, se observa que la presencia de las mayores biomasas de cianobacterias registradas durante el ciclo de muestreo son coincidentes con los mayores niveles de NT, disminuyendo abruptamente sobre el final del periodo (a partir de marzo de 2016), cuando los valores de NT son menores (Figura 5). Por otra parte, la turbiedad (NTU) muestra un importante pico a comienzos de febrero 2016, coincidente con un marcado incremento en las biomasas de cianobacterias, mientras que a partir de marzo de 2016 se dio un importante incremento en NTU acoplado a la caída abrupta de los valores de ficocianina (Figura 5).

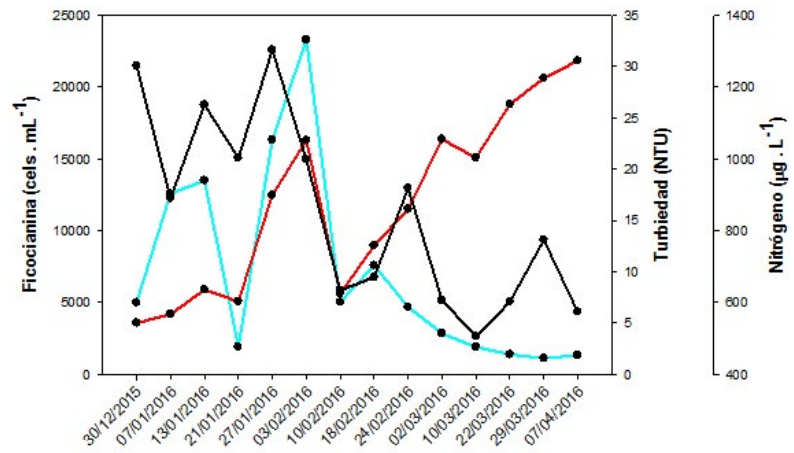

Figura 5. Variación temporal de la ficocianina (color celeste) en cels. $\mathrm{mL}^{-1}$, la turbiedad (color rojo) en NTU y el nitrógeno total (color negro) en $\mu$ g. $\mathrm{L}^{-1}$ para cada una de las fechas de muestreo consideradas.

Considerando los nutrientes NT y PO4, la biomasa de cianobacterias (en términos de ficocianina) presentó una relación positiva respecto al NT (Figura 6A), y negativa respecto al PO4 (Figura 6B).

En cuanto a la significancia del PO4 y NT sobre la composición fitoplanctónica, las diferentes cianobacterias presentes en la laguna durante el periodo de muestreo presentaron patrones de biomasa diferenciales según las concentraciones de estos nutrientes (Figura 7). Los resultados indican que la ocurrencia de las especies de cianobacterias que alcanzaron las mayores biomasas (Dolichospermum crassum y Microcystis aeruginosa) ocurrieron a altos niveles de NT, en el caso de D.crassum en un rango algo mayor de $\mathrm{PO} 4$ en relación a M.aeruginosa, cuyas mayores biomasas se observaron a valores de $\mathrm{PO} 4$ mayores a $20 \mu g . L^{-1}$ (Figura 7A). Por otra parte, otras especies en menores biomasas presentaron respuestas complementarias a los niveles de los nutrientes mencionados (Figura 7C). El complejo Cuspidotthrix issatschenkoii, Aphanizomenon spp y Raphidiopsis $s p p$ presentó biomasas mayores a bajas concentraciones de PO4 y altas de NT (Figura 7D). En cuanto a Sphaerocavum brasiliense, presentó mayores biomasas a valores bajos tanto de PO4 como de NT (Figura 7B), mientras que Dolichospermum
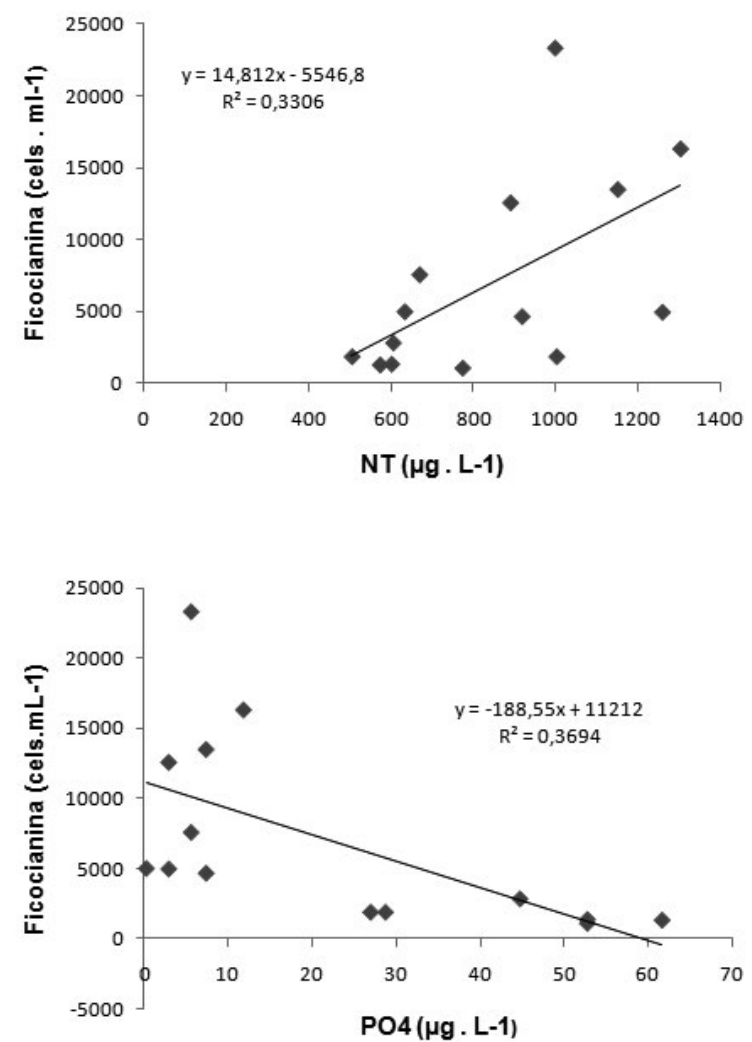

Figura 6. Relación entre la biomasa de cianobacterias (ficocianina) y los nutrientes considerados; A) Nitrógeno total (NT) en $\mu \mathrm{g} . \mathrm{L}^{-1}$ con coeficientes $\mathrm{a}=14,812 \mathrm{y} \mathrm{b}=$ $-5546,8$, con un $\mathrm{R}^{2}=0,3306$ y B) fosfato (PO4) en $\mu \mathrm{g} \cdot \mathrm{L}^{-1}$ con coeficientes $\mathrm{a}=-188,55 \mathrm{y} b=11212$, con un $\mathrm{R}^{2}=0,662$.

solitarium presentó mayores biomasas a valores muy bajos de PO4 e intermedios de NT (Figura 7E).

\section{Discusión}

Laguna del Sauce presenta una alternancia en la biomasa y composición de fitoplancton, con rápidos recambios en periodos muy cortos (una semana). Asimismo, tanto en términos de biomasa como de composición, se observan importantes diferencias entre el sistema principal (puntos: Bahía Mallorquina, Sauce Norte y Sauce Sur) respecto a los sistemas asociados. Esta situación evidencia una alta variabilidad espacio-temporal en el sistema, donde la ocurrencia de floraciones de cianobacterias se restringe al sistema principal, el lugar donde se localiza la toma de agua para potabilización. Además, las floraciones de cianobacterias presentaron un alto recambio en su composición, un patrón de suma relevancia porque la alternancia de especies de cianobacterias presentes (Microcystis aeruginosa, Dolichospermum crassum, Aphanizomenon spp y Cuspidothrix issatschenkoii) estuvo asociada a especies con toxicidad registrada en Uruguay (Bonilla, 2009; De León y Yunes, 2001; Sienra y Ferrari, 2006; Pacheco, et al., 2016). Esta es una situación de riesgo considerando las altas biomasas alcanzadas particularmente por M.aeruginosa y D.crassum, y el uso para potabilización de este ecosistema. En todos los casos, las floraciones de 
A

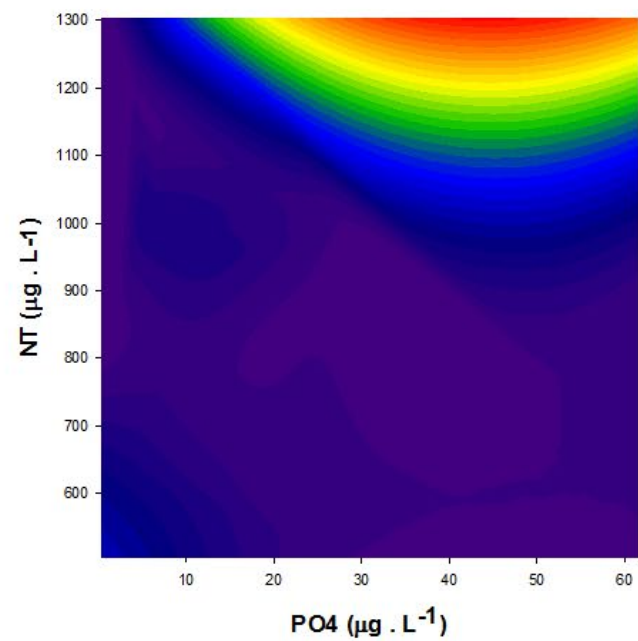

C
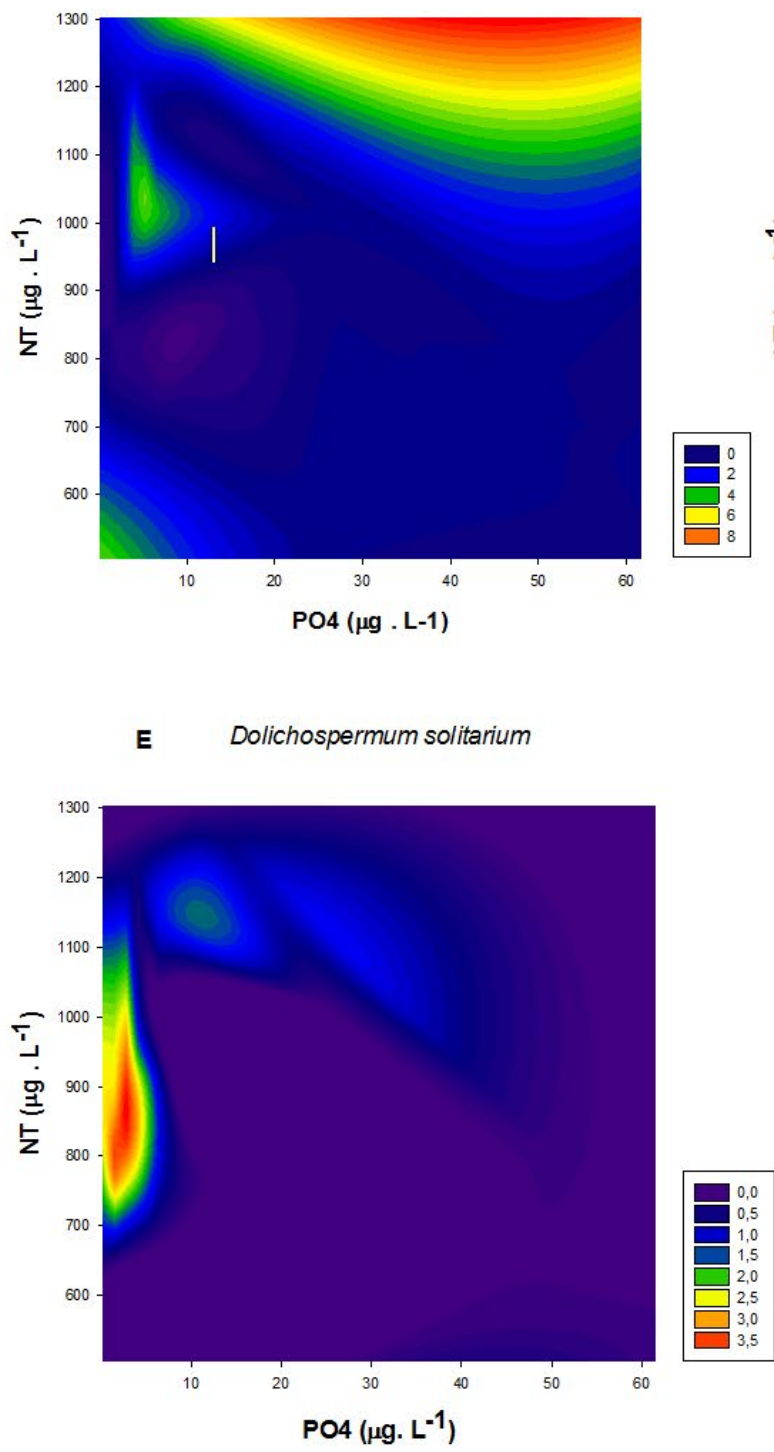

B

Sphaerocavum brasiliense
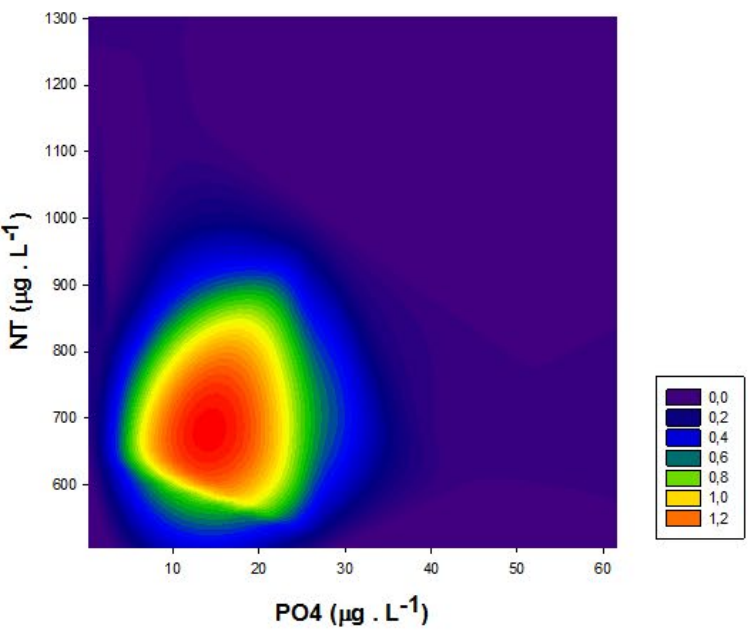

D Cuspidothrix spp / Aphanizomenon spp / Raphidiopsis spp

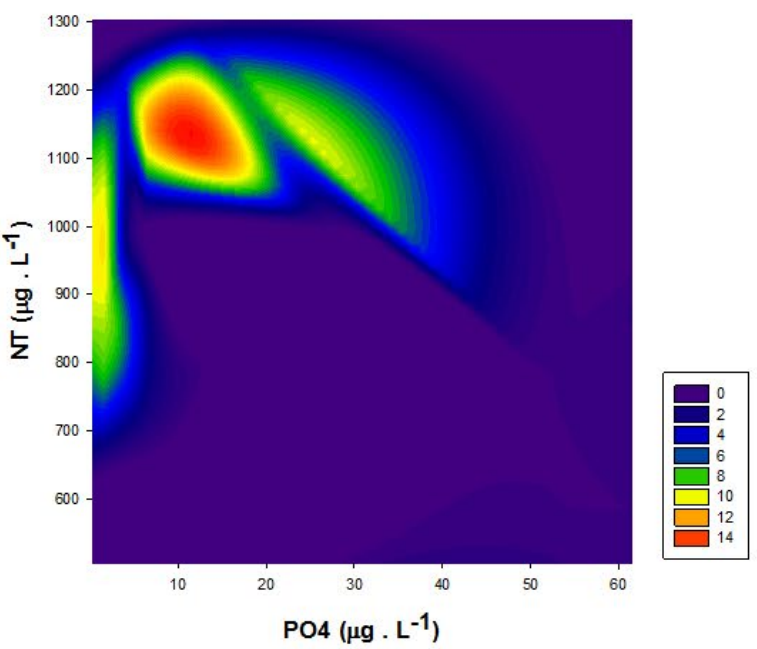

Figura 7. Gráficos de densidad que indican el biovolumen de cada grupo de cianobacterias en relación a la conjunción NT vs PO4. Los colores indican los valores de biomasa registrados. El color rojo corresponde a las máximas biomasas registradas y el violeta a las mínimas. 
cianobacterias presentes en el cuerpo de agua principal se encontraron en biovolúmenes muy superiores al máximo nivel de alerta en agua bruta para potabilización, lo cual indicaría un riesgo sumamente alto en caso de que ingresara al sistema de tratamiento (Gravier, et al., 2017).

Esta alternancia de altos biovolúmenes de cianobacterias de potencial tóxico plantea un escenario de manejo muy complejo, ya que los recambios composicionales ocurren en periodos muy cortos de tiempo y comprenden una escala espacial muy amplia. Esto limita la capacidad de anticiparse al desarrollo de las floraciones basándose en incrementos progresivos de determinadas especies o grupos de riesgo potencial.

Por otra parte, se encontró que las floraciones de cianobacterias, registradas primero en el cuerpo de agua principal, luego de desaparecer de este persistían en los sistemas aledaños en biomasas menores. Esto sugiere que dichos subsistemas podrían actuar como reservorios de floraciones de cianobacterias precedentes en el sistema principal, pudiendo actuar luego como inóculos para futuras floraciones en ese sistema.

Los resultados de este estudio indican que los principales factores estructuradores del fitoplancton en Laguna del Sauce son: la turbiedad del sistema, que estaría actuando como un regulador de la biomasa de fitoplancton por limitación lumínica, pero que a su vez se ve influenciado por ella, y los niveles de NT y PO4, como principales estructuradores de la composición del fitoplancton y en particular de las especies de cianobacterias detectadas. Así, la disminución abrupta de las biomasas de cianobacterias en Laguna del Sauce estaría vinculada a un aumento en la turbiedad (NTU) y a una caída abrupta en el NT. Estos resultados son en parte coincidentes con lo evidenciado por Crisci et al. (en prensa) en cuanto al rol de la turbiedad como limitante del desarrollo de alta biomasa fitoplanctónica en Laguna del Sauce. Sin embargo, todos los valores de turbiedad registrados en este estudio son inferiores el límite descrito anteriormente de 30NTU (Crisci, et al., en prensa). Los resultados de nuestro estudio indicarían un rol conjunto de regulación de la biomasa de cianobacterias en el sistema, donde si bien los niveles de turbiedad no son superiores a los 30NTU, la limitante de NT actuaría en conjunto con la turbiedad limitando el desarrollo de altas biomasas de cianobacterias en el sistema.

Por otra parte, si bien la composición fitoplanctónica en general estaría regulada por múltiples variables ambientales, en el caso de las cianobacterias su composición estaría mayormente determinada por la conjunción de las concentraciones NT:PO4.

Estudios previos realizados para lagos en Alemania por Dolman et al. (2012) indican que las diferentes especies de cianobacterias presentan respuestas diversas a las relaciones NT:PT, demostrando que ni todas las cianobacterias en su conjunto, ni las cianobacterias nostocales (que comparten la capacidad de fijación de N2 atmosférico) deben ser consideradas como un grupo homogéneo en sus requerimientos de estos nutrientes, y que las relaciones entre estos nutrientes son clave para predecir la presencia de determinadas especies de cianobacterias. En este sentido, los resultados que aquí se presentan indican un patrón diferencial de la composición de especies de cianobacterias en función de las relaciones NT:PO4, concordante con lo registrado por Dolman et al. (2012). Particularmente, cabe destacar que las especies presentes en altas biomasas, generadoras de floraciones en Laguna del Sauce: Microcystis aeruginosa y Dolichospermum crassum, poseen requerimientos similares de altos niveles de NT y un mínimo de $20 \mu \mathrm{g} . \mathrm{L}^{-1}$ de PO4 para desarrollar altas biomasas. Esto es de suma relevancia dadas las diferencias funcionales en cuanto a la capacidad de fijación de N2 entre $M$. aeruginosa (Chroococcal) y D. crassum (Nostocal). Con base en estos resultados se destaca la importancia en el control de los niveles de NT en el sistema, lo cual podría prever la aparición de altas biomasas de estas especies. En este sentido, Conley et al. (2009) subrayan la importancia de controlar la entrada de ambos nutrientes ( $\mathrm{N}$ y $\mathrm{P}$ ) para controlar la eutrofización y, en consecuencia, sus efectos adversos asociados (floraciones de cianobacterias).

Estos resultados indican el rol clave de los nutrientes como factores ambientales asociados a la composición de fitoplancton en Laguna del Sauce y confirman el rol de la turbiedad como regulador de la biomasa fitoplanctónica en el sistema. En particular, se destaca el rol del NT sobre la biomasa de cianobacterias y las relaciones NT:PO4, vinculados a la presencia diferencial de especies de cianobacterias potencialmente tóxicas. Los resultados de este trabajo son clave para el entendimiento de los procesos vinculados al fitoplancton en el ecosistema. Considerando el uso de la Laguna del Sauce como fuente de agua para potabilización, estos resultados pueden contribuir al desarrollo de indicadores fisicoquímicos sencillos basados en niveles de NTU y NT:PO4 que permitan predecir la presencia de diferentes especies de cianobacterias.

\section{Referencias}

Bonilla, S., Haakonson, S., Somma, A., Gravier, A., Britos, A., Vidal, L., De León, L., Brena, B., Pírez, M., Piccini, C., Martínez de la Escalera, G., Chalar, G., González-Pianna, M., Martigani, F. y Aubriot, L., 2015. Cianobacterias y cianotoxinas en ecosistemas límnicos de Uruguay. En: INNOTEC, 10, pp.9-22.

Bonilla, S., 2009. Cianobacterias planctónicas del Uruguay. Manual para la identificación y medidas de gestión. Montevideo: UNESCO; PSHI-VII. (Documento técnico, 16). ISBN 978-92-9089-138-3.

Carmichael, W.W., 1992. Cyanobacteria secondary metabolites-the cyanotoxins. En: Journal of applied bacteriology, 72(6), pp.445-459.

Carmichael, W.W., 1997. The cyanotoxins. En: Advances in Botanical Research, 27, pp.211-256.

Carpenter, S.R., Stanley, E.H. y Vander Zanden, M.J., 2011. State of the world's freshwater ecosystems: physical, chemical, and biological changes. En: Annual Review of Environment and Resources, 36, pp.75-99.

Chorus, I., 2012. Cyanotoxins: occurrence, causes, consequences. Berlín: Springer. ISBN-13: 978-3-642-64004-9.

Chorus, I. y Bartram, J., 1999. Toxic cyanobacteria in water: A guide to their public health consequences, monitoring and management. Londres: E \& FN Spon. ISBN: 0419239308.

Conley, D.J., Pearl, H.W., Howarth, R.W., Boesch, D.F., Seitzinger, S.P., Havens, K.E., Lancelot, C. y Likens, G.E., 2009. Controlling Eutrophication: Nitrogen and Phosphorus. En: Science, 232, pp.1014-1015.

Crisci, C., Terra, R., Bidegain, M., Ghattas, B., Goyenola, G., Lagomarsino, J.J., M., Méndez, G., Pacheco, J.P. y Mazzeo, N., en prensa. Multi-model approach to predict phytoplankton biomass and composition dynamics in a eutrophic shallow lake. En: Ecological Modelling.

De León, L. y Yunes, J., 2001. First report of a microcystincontaining bloom of the cyanobacterium microcystis 
aeruginosa in the La Plata River, South America. En: Enviromental Toxicology, 16(1), pp.110-112.

De León, L., 2002. Floraciones de cianobacterias en aguas continentales de Uruguay: causas y consecuencias. En: Domínguez, A. y Prieto, R.G., eds. Perfil ambiental del Uruguay, 2002. Montevideo: Nordan-Comunidad. pp.28-37. Dolman, A.M., Rücker, J., Pick, F.R., Fastner, J., Rohrlack, T., Mischke, U. y Widner, C., 2012. Cyanobacteria and Cyanotoxins: The Influence of Nitrogen versus Phosphorus. En: PLoS ONE, 7(6): e38757. doi:10.1371/ journal.pone.0038757.

Falconer, I.R., Burch, M.D., Steffensen, D.A., Choice, M. y Coverdale, O.R., 1994. Toxicity of the blue-green alga (cyanobacterium) Microcystis aeruginosa in drinking water to growing pigs, as an animal model for human injury and risk assessment. En: Environmental Toxicology, 9(2), pp.131-139.

Falconer, I.R. y Humpage, A.R., 2005. Health risk assessment of cyanobacterial (blue-green algal) toxins in drinking water. En: International Journal of Environmental Research and Public Health, 2(1), pp.43-50.

Ferber, L.R., Levine, S.N., Lini, A. y Livingston, G.P., 2004. Do cyanobacteria dominate in eutrophic lakes because they fix atmospheric nitrogen? En: Freshwater Biology, 49(6), pp.690-708.

Guillard, R.L., 1978. Counting slides. En: Sournia, A., ed., 1978. Phytoplankton manual. Paris: Unesco. pp.182-190.

Gravier, A., Britos, A., Vidal, L. y Pérez, M.C., 2017. Protocolo de alertas por cianobacterias en los servicios de agua potable de OSE. Montevideo: OSE. (Documento Técnico).

Heisler, J., Gilbert, P.M, Burkholder, J.M., Anderson, D.M., Cochlan, W., Dennison, W.C., Dortch, Q., Gobler, C.J., Heil, C.A., Humphries, E., Lewitus, A., Magnien, R., Marshall, H.G., Sellner, K., Stockwell, D.A., Stoecker, D.K. y Suddleson, M., 2008. Eutrophication and harmful algal blooms: A scientific consensus. En: Harmful Algae, 8, pp.3-13.

Hillebrand, H., Durselen, C.D., Kirschtel, D., Pollingher, U. y Zohary, T., 1999. Biovolume calculation for pelagic and benthic microalgae. En: Journal of Phycology, 35, pp.403-424

Hutchinson, G., 1973. Eutrophication. En: American Science, 61, pp. $269-279$

Inda, H. y Steffen, M., eds., 2010. Bases técnicas para el manejo integrado de Laguna del Sauce y cuenca asociada. Montevideo: Editorial Montevideo. ISBN: 978-9974-0-0694-2.

Khan, F. y Ansari, A., 2005. Eutrophication: an ecological vision. En: The Botanical Review, 71(4), pp.449-482.

Koroleff, F., 1970. Revised version of direct determination of amonia of natural waters as indophenol blue. Int. Con. Explor. Sea C.M 1969/C9. ICES, Information on techniques and methods for sea water analysis. En: Interlab. Rep., 3, pp.19-22.

Lund, J., Kipling, C. y Le Cren, E., 1958. The inverted microscope method of estimating algal numbers and statistical basis of estimating by counting. En: Hydrobiologia, 11, pp.143-170.

Mazzeo, N., García-Rodríguez, F., Rodríguez, A., Méndez, G., Iglesias, C., Inda, H., Goyenola, G., García, S., Fosalba, C., Marroni, S., Crisci, C., Del Puerto, L.;,Clemente, J., Pacheco, P., Carballo, C., Kroeger, A., Vianna, M., Meerhoff, M., Steffen, M., Lagomarsino, J.J., Masdeu, M., Vidal, N., Texeira de Mello, F., González, I. y Larrea, D., 2010. Estado trófico de Laguna del Sauce y respuestas asociadas. En: Inda, H. y Steffen, M., eds., 2010. Bases técnicas para el manejo integrado de Laguna del Sauce y cuenca asociada. Montevideo: Editorial Montevideo. pp.31-49. ISBN: 978-9974-0-0694-2.

Merel, S., Walker, D., Chicana, R., Snyder, S., Baurès, E. y Thomas, O., 2013. State of knowledge and concerns on cyanobacterial blooms and cyanotoxins. En: Environment international, 59, pp.303-327.

Moss, B., Madgwick, J. y Phillips, G., 1996. A guide to the restoration of nutrient enriched shallow lakes. Norwich: Environmental Agency. ISBN: 0948119292.

Muller, R. y Wiedemann, O., 1955. Die bestimmung des nitrations in Wasser. En: Von Wasser, 22, pp.247-271.

Murphy, J. y Rilley, J.P., 1962. A modified single-solution method for the determination of phosphate in natural waters. En: Analyt. Chim. Acta, 27, pp.31-36.

Nusch, E., 1980. Comparisons of different methods for chlorophyll and phaeopigments determination. En: Archiv für Hydrobiologie Ergebnisse der Limnologie, 14, pp.14-36.

Pacheco, J.P., Iglesias, C., Meerhoff, M., Fosalba, C., Goyenola G., Teixeira-de Mello, F., García, S., Gelós, M. y GarcíaRodríguez, F., 2010. Phytoplankton community structure in five subtropical shallow lakes with different trophic status (Uruguay): a morphology-based approach. En: Hydrobiologia, 646(1), pp.187-197.

Pacheco, J.P., González-Madina, L., Clemente, J.M. y Mazzeo, N., 2016. Análisis cualitativo y cuantitativo del fitoplancton de la Laguna del Sauce Maldonado - Uruguay. Montevideo: OSE, UGD. (Informes hidrobiológicos semanales de calidad de agua).

Paerl, H.W., 1997. Coastal eutrophication and harmful algal blooms: importance of atmospheric deposition and groundwater as «new» nitrogen and other nutrient sources. En: Limnology and Oceanography, 42, pp.1154-1165.

Paerl, H.W., Hall., N.S. y Calandrino., E.S., 2011. Controlling harmful cyanobacterial blooms in a world experiencing anthropogenic and climate-induced change. En: Science of the Total Environment, 409(10), pp.1739-45.

Paerl, H.W. y Otten, T.G., 2013. Harmful cyanobacterial blooms: Causes, consequences and controls. En: Microbial Ecology, 65(4), pp.995-1010.

Prepas, E. y Charette T., 2003. Worldwide eutrophication of water bodies: causes, concerns, controls. En: Sherwood, B., ed. Treatise on geochemistry. Vol. 9. Amsterdam Elsevier. pp.311-331.

Rodríguez, A., Méndez, G., Kausas, S., Clemente, J., Kroger, A. y Mazzeo, N., 2010. Importancia de la carga externa e interna de nutrientes en el estado trófico de la Laguna del Sauce. En: Inda, H. y Steffen, M., eds., 2010. Bases técnicas para el manejo integrado de Laguna del Sauce y cuenca asociada. Montevideo: Editorial Montevideo. pp.53-61. ISBN: 978-9974-0-0694-2.

Reynolds, C.S., 1987. Cyanobacterial water-blooms. En: Advances in Botanical Research, 13(C), pp.67-143.

Reynolds, C.S., 2006. The ecology of phytoplankton. Cambridge: Cambridge University Press.

Robarts, R. y Zohary, T., 1987. Temperature effects on photosynthetic capacity, respiration and growth rates of bloom-forming cyanobacteria. En: New Zealand Journal of Marine and Freshwater Research, 21, pp.391-399.

Schindler, D.W., 2006. Recent advances in the understanding and management of eutrophication. En: Limnology and Oceanography, 51(1), pp.356-363 
Schindler, D.W., Hecky, R.E., Findlay, D.L., Stainton, M.P., Parker, B.R., Paterson, M.J., Beaty, K.G., Lying, M. y Kasian, S.E.M., 2008. Eutrophication of lakes cannot be controlled by reducing nitrogen input: Results of a 37-year wholeecosystem experiment. En: PNAS, 105(32), pp.11254-11258

Sienra, D. y Ferrari, G., 2006. Monitoreo de cianobacterias en la costa de Montevideo. En: Menafra, R.; Rodriguez-Gallego, L.; Scarabino, F. y Conde, D., eds., 2006. Bases para la conservación y el manejo de la costa uruguaya. Montevideo: Vida Silvestre. pp.413-420. ISBN: 9974-7589-2-0.

Smith, V. y Bennett, S., 1999. Nitrogen:Phosphorus supply ratios and phytoplankton community structure in lakes. En: Archiv fur Hydrobiologie, 146, pp.37-53.

Smolders, A.J.P., Lamers, L.P.M., Lucassen, E.C.H.E.T., Van Der Velde, G. y Roelofs, J.G.M., 2006. Internal eutrophication: How it works and what to do about it $-\mathrm{a}$ review. En: Journal of chemistry and ecology, 22, pp.93-111.

Stewart, I., Webb, P., Schluter, P. y Shaw, G., 2006. Recreational and occupational field exposure to freshwater cyanobacteria -a review of anecdotal and case reports, epidemiological studies and the challenges epidemiologic assessment. En: Environmental Health: A Global Access Science Source, 5(6), pp.1-13.
Tarczynska, M., G. Nalecz-Jawecki, Z. Romanowska-Duda, J. Sawicki, K. Beattie, G. Codd y Zalewski., M., 2001. Tests for the toxicity assessment of cyanobacterial bloom samples. En: Environ. Toxicol., 16, pp.383-390.

Uhelinger, V., 1964. Étude statistique des méthodes de dénobrement planctonique. En: Archive de Science, 17, pp.121-223.

Utermöhl, H., 1985. Zur vervolkommung der quantitativen phytoplankton-methodik. mitteilungen. En: Internationale Vereiningung fuer Theoretische and Angewandte Limnologie, 9, pp.1-38.

Valderrama, J.C., 1981. The simultaneous analysis of total $\mathrm{N}$ and total P in natural waters. En: Mar. Chem., 10, pp.1009-1022.

Vidal, L. y Kruk, C., 2008. Cylindrospermopsis raciborskii (Cyanobacteria) extends its distribution to Latitude 34 53'S: taxonomical and ecological features in Uruguayan eutrophic lakes. En: Panamerican Journal of Aquatic Sciences, 3, pp.142-151.

Wetzel, R.G., 2001. Limnology: Lake and river ecosystems. San Diego: Academic Press. ISBN-13: 978-0-12-744760-5.

WHO, 2004. Guidelines for drinking-water quality. Vol. 1. Singapore: World Health Organization. ISBN: 9241546743. 